\title{
Optimum sol viscosity for stable electrospinning of silica nanofibres
}

Jozefien Geltmeyer ${ }^{1}$, Lien Van der Schueren ${ }^{1}$, Frederik Goethals ${ }^{2}$, Klaartje De Buysser $^{2}$, Karen De Clerck ${ }^{1}$

${ }^{1}$ Fibre and Colouration Technology Research Group, Department of Textiles, Faculty of Engineering and Architecture, Ghent University, Technologiepark 907, 9052 Zwijnaarde (Ghent), Belgium

${ }^{2}$ Sol Gel Centre for Research on Inorganic Powder and Thin Film Synthesis, Department of Inorganic and Physical Chemistry, Faculty of Sciences, Ghent University, Krijgslaan 281 S3, 9000 Ghent, Belgium

Corresponding author: Karen.DeClerck@UGent.be

Tel. +3292645740

Fax. +3292645846

Silica nanofibres have, due to their excellent properties, promising characteristics for multiple applications such as filtration, composites, catalysis, etc. Silica nanofibres can be obtained by combining electrospinning and the sol-gel process. To produce silica nanofibres most of the time organic solutions are applied containing a carrying polymer, which is afterwards removed by a thermal treatment to form pure ceramic nanofibres. Although electrospinning of the pure silica precursors without carrying polymer is preferred, the parameters influencing the stability of the electrospinning process are however largely unknown. In addition this knowledge is essential for potential up-scaling of the process. In this study, the optimum viscosity to electrospin in a stable manner is determined and the way to obtain this viscosity is evaluated. Sols with a viscosity between 120 and $200 \mathrm{mPa}$.s could be electrospun in a stable way, resulting in uniform and beadless nanofibres. Furthermore, this viscosity region corresponded with nanofibres having the lowest mean nanofibre diameters. Electrospinning with diluted sols was possible as well, but electrospinning of the fresh sols was more stable. These results illustrate the importance of the viscosity and degree of crosslinking of the sol for the stable electrospinning of silica nanofibres and demonstrate that upscaling of the electrospinning process of silica nanofibres is feasible.

electrospinning, sol-gel, stability, silica, tetraethyl orthosilicate

\section{Introduction}

Electrospinning is an efficient technique using electrostatic forces to obtain polymer and ceramic nanofibres [1]. An electric field is applied between the tip of a nozzle, through which the solution is flowing, and a grounded collector plate. The electrostatic forces distort the solution from a spherical pendant drop to a Taylor cone [2]. Once the electrostatic forces overcome the surface tension, a charged jet is ejected, which is subsequently elongated due to bending and 
splaying. As a consequence a non-woven structure of continuous fibres with diameters ranging from a few nanometres to several microns is deposited on the collector plate. Electrospinning of polymer nanofibres has been studied extensively [1-3] and has shown to be possible via stable, reproducible and controllable processes [4-7]. Steady state electrospinning for polymers is reported as a solution for obtaining a high stability and reproducibility [4]. Electrospinning reaches steady state when the amount of polymer transported through the needle per time unit equals the amount of polymer deposited as nanofibres on the collector per time unit. Steady state electrospinning requires a constant stable Taylor cone as a function of time. This guarantees beadless nanofibres and a high reproducibility. In addition, the nanofibres are to be deposited on a well-defined area below the nozzle, since this allows for a uniform thickness of the non-woven in future upscaling.

Studies on ceramic nanofibres are less comprehensive. Ceramic materials are hard and inert and are therefore known for their excellent properties such as high temperature resistance and chemical inertness. These promising characteristics allow ceramic nanofibres to be used for various applications such as biological applications [8,9], filtration [10,11], composites [12,13], catalysis [14], etc.

Two approaches can be used for obtaining ceramic nanofibres by electrospinning. The use of an aqueous solution made from alkoxide precursors such as tetraethyl orthosilicate (TEOS) is a possible synthesis route. Secondly, an organic solution containing alkoxide precursors and carrying polymers can be applied [15-18]. The latter is most frequently applied because the rheological properties of polymer solutions are more convenient to adjust and control [18]. After electrospinning the polymer component is mostly removed by a thermal treatment to form pure ceramic nanofibres. Due to this thermal treatment the fibre surface becomes rough and uneven and the fibres exhibit poor mechanical properties [19]. Although being more challenging, electrospinning of pure alkoxide precursors is thus preferred.

The electrospinning of alkoxide precursors is not well understood yet, with only a few recent published studies [8,20-23]. No detailed literature on the influence of the sol properties on the resulting ceramic nanofibres is available. In addition, no attention has been given to the stability of the electrospinning process. This knowledge, however, showed to be a crucial prerequisite for polymeric nanofibres 
and is thus essential for future practice of silica nanofibres as well. Iimura et al. [20] and $\mathrm{Xu}$ et al. [23] determined that TEOS sols are only spinnable when a linear organization of the silica tetrahedra is obtained. This linearity is influenced by the initial amount of water and acid, making these important parameters of the process. One of the characteristics that highly affects the electrospinning process is the viscosity. This viscosity is caused by the degree of crosslinking which is in its turn influenced by several factors such as heating time, temperature, the amount of water and acid. To obtain a desired viscosity, dilution of the sol solution after evaporation is also possible. The influence hereof on the electrospinning process will be determined.

A detailed study on the electrospinning of silica nanofibres is vital. This paper focuses on the electrospinning of TEOS. By selecting one precursor a constant reactivity of the siloxane precursor is ensured, and the influence hereof on the viscosity and the spinnability is thus excluded. The viscosity range that yields uniform and reproducible nanofibres is determined, followed by an analysis of the fibre morphology. Next, the influence of dilution of the sol electrospinning solutions on the resulting nanofibres is established, to determine whether only the viscosity or also the way to obtain this viscosity has an influence. Since no research has been carried out on the stability of the electrospinning process of TEOS, the stability of the Taylor cone will be evaluated in this study as a first step towards a stable and reproducible electrospinning process. The results of this paper may contribute to a future breakthrough of ceramic nanofibres.

\section{Materials and methods}

The sol-gel precursor tetraethyl orthosilicate (TEOS, reagent grade 98\%) was obtained from Sigma-Aldrich and used as received. The catalyst, hydrochloric acid $(\mathrm{HCl}, 37 \%)$ and solvent, absolute ethanol, were also supplied by SigmaAldrich. The sols used for electrospinning were prepared by modifying the procedure reported by Choi et al [21]. The silica sol was prepared from a mixture of TEOS, ethanol, distilled water and $\mathrm{HCl}$ at molar ratios of 1:2:2:0.01. First, TEOS was mixed with ethanol. Secondly, aqueous $\mathrm{HCl}$ solution was added to the TEOS/ethanol solution under vigorous stirring with a magnetic stir bar. This solution was heated under stirring at $80^{\circ} \mathrm{C}$ until the volume decreased to approximately $3 / 8$ of the initial volume and the desired viscosity was reached. 
Finally the solution was cooled down to room temperature resulting in a viscous sol.

During the electrospinning process the sol was loaded in a $20-\mathrm{mL}$ plastic syringe equipped with a needle with an inner diameter of $1.024 \mathrm{~mm}$. The flow rate of the sol was regulated with a syringe KD Scientific Pump Series 100 and a Glassman High Voltage Series EH 30P3 source was used to apply a voltage of $22.5 \mathrm{kV}$. The nanofibres were collected on a grounded aluminium foil. All electrospinning experiments were performed at room temperature $\left(21 \pm 1^{\circ} \mathrm{C}\right)$ and room humidity $(33 \pm 10 \% R H)$. The tip to collector distance was fixed at $15 \mathrm{~cm}$ and the flow rate was set at $1 \mathrm{~mL} \mathrm{~h}^{-1}$. Samples with a viscosity lower than $100 \mathrm{mPa}$.s were electrospun at a flow rate of $2 \mathrm{~mL} \mathrm{~h}^{-1}$.

The stability of the electrospinning process was evaluated by visually evaluating the Taylor cone in time and by verifying the absence of droplets and beads on the SEM images.

The viscosity of the solutions was measured prior to electrospinning using a Brookfield viscometer LVDV-II. Fibre morphology and fibre diameters were examined with SEM (Jeol Quanta 200 F FE-SEM) at an accelerating voltage of 20 $\mathrm{kV}$. Before SEM analysis the samples were coated with gold using a sputter coater (Balzers Union SKD 030). The diameter of the nanofibres were measured using Olympus Cell D software by taking an average of 50 measurements.

The Fourier Transform Infrared (FTIR) spectra of the nanofibres were recorded on a Perkin-Elmer GX 2000 in the range $600-4000 \mathrm{~cm}^{-1}$ with a resolution of $4 \mathrm{~cm}^{-1}$ and a data interval of $1 \mathrm{~cm}^{-1}, 16$ scans were taken for each measurement.

\section{Results and discussion}

\section{Determination of electrospinnable viscosity range}

The viscosity plays a dominant role when analysing the electrospinnability of solutions [3,24]. A key prerequisite for obtaining reproducible electrospun nanofibrous non-wovens, is the presence of a stable Taylor cone [3]. In this study sols with different viscosities were attempted to electrospin and were visually evaluated on the presence of a stable Taylor cone. Sols with varying viscosities were obtained by changing the heating time of the sols at $80^{\circ} \mathrm{C}$. During the heating process, the ethanol evaporates and the TEOS started to crosslink due to 
hydrolysis and condensation, thus increasing the viscosity of the solution. It should be noted that the evaporation of ethanol will not only cause a change in viscosity, but also the relative concentrations of the components will change. However, the viscosity is chosen as parameter to characterize the sols after preparation.

As a second prerequisite, the stability of the viscosity during electrospinning was determined. It was found that the viscosity remained constant at minimum during one hour after preparation of the sol.

Different viscosities were obtained, which had varying spinnabilities (Figure 1). The spinnability at each viscosity is expressed with a number from 1 to 5 (Table 1). No stable Taylor cone was obtained for the samples with a spinnability 1 and 2. No nanofibres could be obtained for spinnability 1 . Spinnability 2 corresponds with nanofibres having a lot of beads and droplets. Starting from spinnability 3 a stable Taylor cone could be obtained with increasing time period. Spinnability 4 and 5 are defined as stable electrospinning. However, for each spinnability above spinnability 2 instabilities are seen after the time mentioned in Table 1 . These instabilities shown by the Taylor cone are visualised in Figure 2. A clear difference can be seen between a stable Taylor cone and an unstable Taylor cone. It should be noticed that this unstable Taylor cone recovered after removal of the solidified piece and thus became stable again.

Table 1 Explanation of the different spinnabilities

\begin{tabular}{ll}
\hline & Spinnability \\
\hline 1 & Not spinnable \\
2 & Spinnable with drops \\
3 & Spinnable with a stable Taylor cone for 30s \\
4 & Spinnable with a stable Taylor cone for 1 min \\
5 & Spinnable with a stable Taylor cone for 3-5 min \\
\hline
\end{tabular}




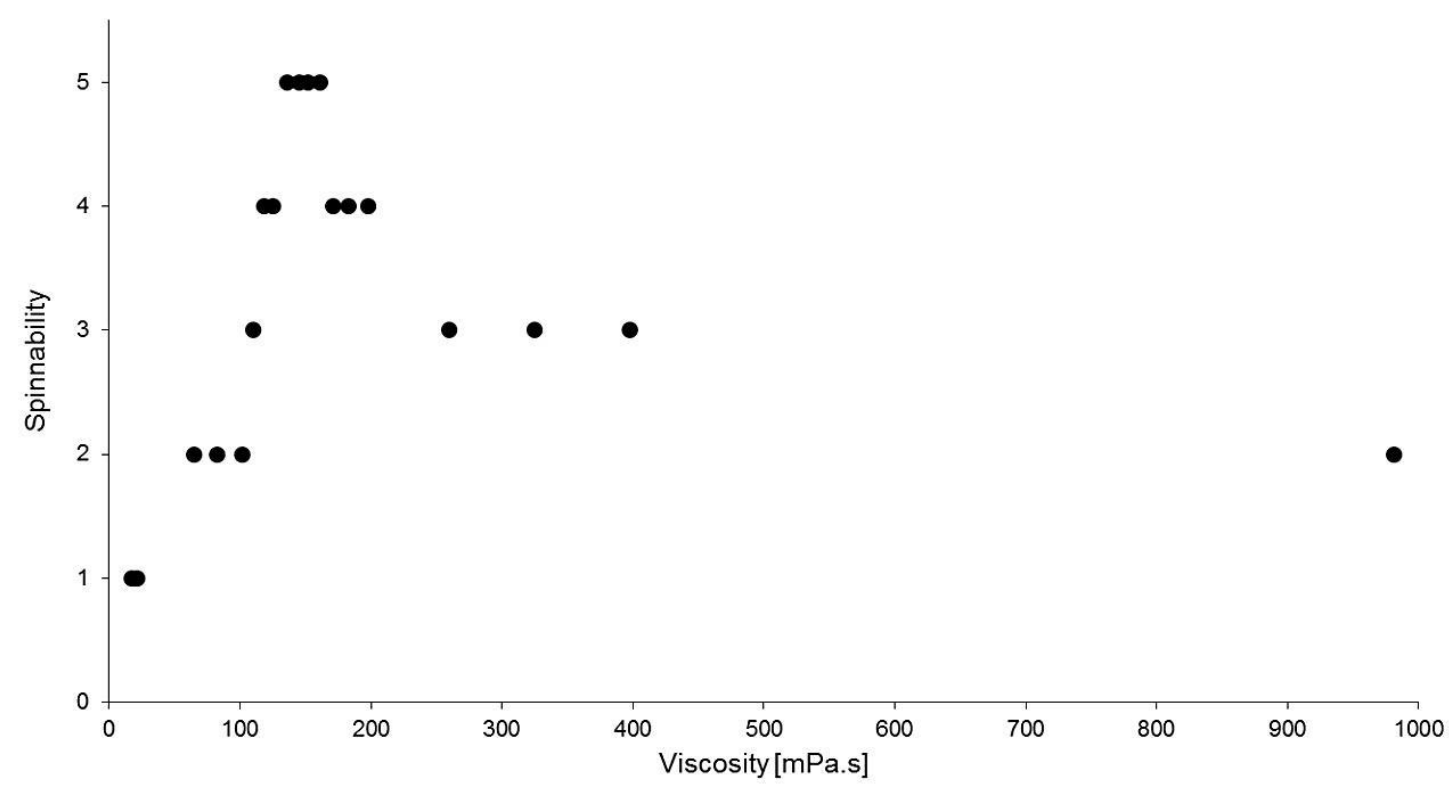

Fig. 1 Spinnability as a function of the sol viscosity
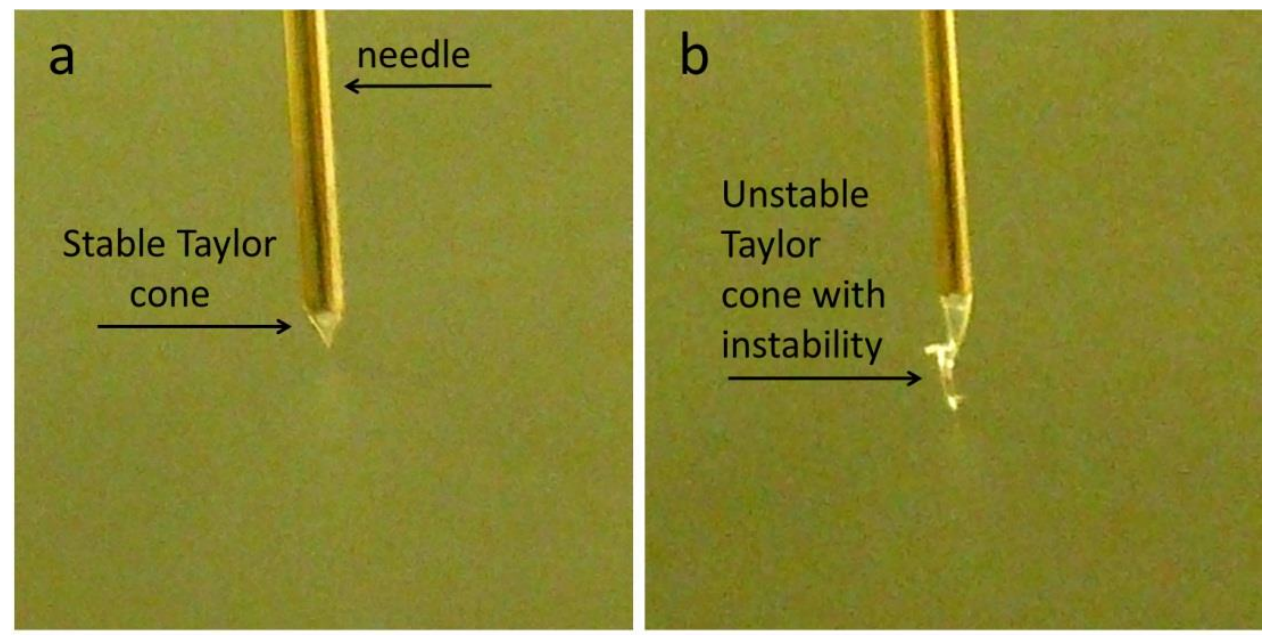

Fig. 2 Stable Taylor cone (a) and unstable Taylor cone (b)

Nanofibres were collected on the collector plate starting from a viscosity of 65 mPa.s up to a viscosity of about $1000 \mathrm{mPa}$.s. When the viscosity was lower than $100 \mathrm{mPa} . \mathrm{s}$, beads and droplets were clearly visible in the SEM images of the samples (Figure $3(\mathrm{a}, \mathrm{b})$ ) and stable electrospinning was not possible. Further increasing the viscosity resulted in more stable electrospinning, which is confirmed by the uniform, beadless nanofibres in the SEM images (Figure 3 (cg)). A clear viscosity window, between 130 and $160 \mathrm{mPa}$.s, was observed in which electrospinning was most stable having spinnability 5 and showing a stable Taylor cone for the longest period of time (Figure 1). Yet, a broader viscosity 
window can be determined between 120 and $200 \mathrm{mPa}$.s having spinnability 4, also showing stable electrospinning behaviour. It is to be noted that despite the instabilities of the Taylor cone, all samples resulting from solutions with a viscosity starting from $110 \mathrm{mPa}$.s to $981 \mathrm{mPa}$.s, corresponding with spinnability 3 and higher, showed uniform, beadless fibres in the SEM images. However, the sample with a viscosity of $981 \mathrm{mPa} . \mathrm{s}$ showed no stable electrospinning and the fibres were not as uniform as the fibres obtained at lower viscosities, Figure 3(h).

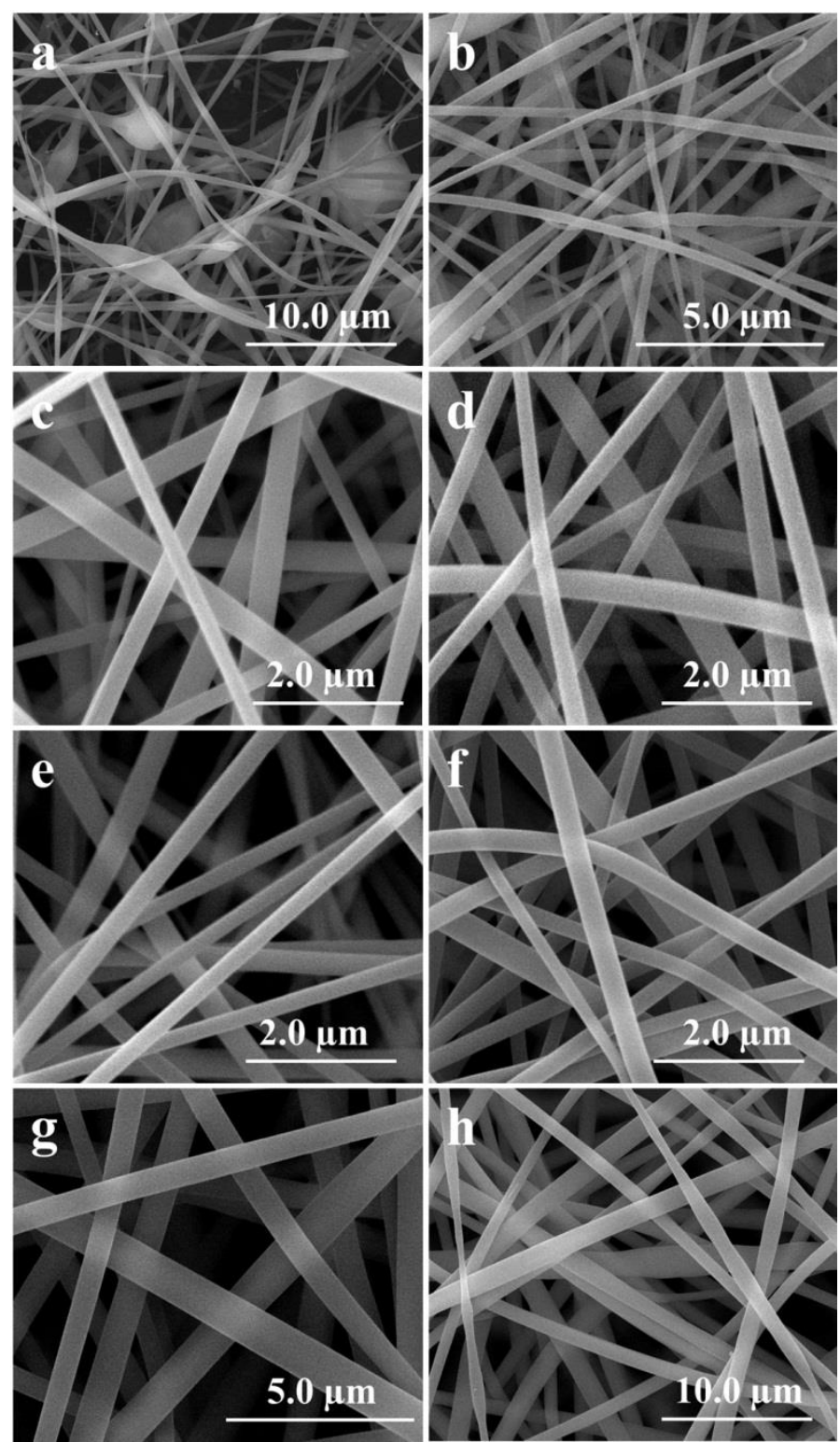

Fig. 3 SEM images electrospun silica nanofibres (a: 65 mPa.s, b:102 mPa.s, c:110 mPa.s, d:125 mPa.s, e:145 mPa.s, f:198 mPa.s, g:398 mPa.s, h:981 mPa.s)

A clear influence of the viscosity on the nanofibre diameter can be noticed (Figure 4). A minimum viscosity is necessary to obtain a stable Taylor cone and uniform, beadless nanofibres. Reaching this minimum viscosity results in a decrease in 
nanofibre diameter. Furthermore, the lowest mean nanofibre diameters are obtained for the samples in the stable electrospinning region. These samples have a nearly constant nanofibre diameter in between $210 \mathrm{~nm}$ and $310 \mathrm{~nm}$. Moreover, when having a broader look to the samples with a spinnability of 3 as well, an increase in nanofibre diameter is observed with an increase in solution viscosity. The fibre diameter increases from $260 \pm 70 \mathrm{~nm}$ at a viscosity of $136 \mathrm{mPa} . \mathrm{s}$ to 670 $\pm 200 \mathrm{~nm}$ at a viscosity of $398 \mathrm{mPa} . \mathrm{s}$. This is in line with electrospinning of polymer solutions, where an increase in viscosity also results in an increasing fibre diameter [24,25]. When the viscosity of a solution rises, a higher resistance of the jet to the bending instability and a faster solidification of the jet, indeed lead to thicker fibres [6,26]. Typical variation coefficients are between $20 \%$ and $30 \%$. These is in line with variation coefficients of polymer nanofibres found in literature, such as polycaprolactone [5].

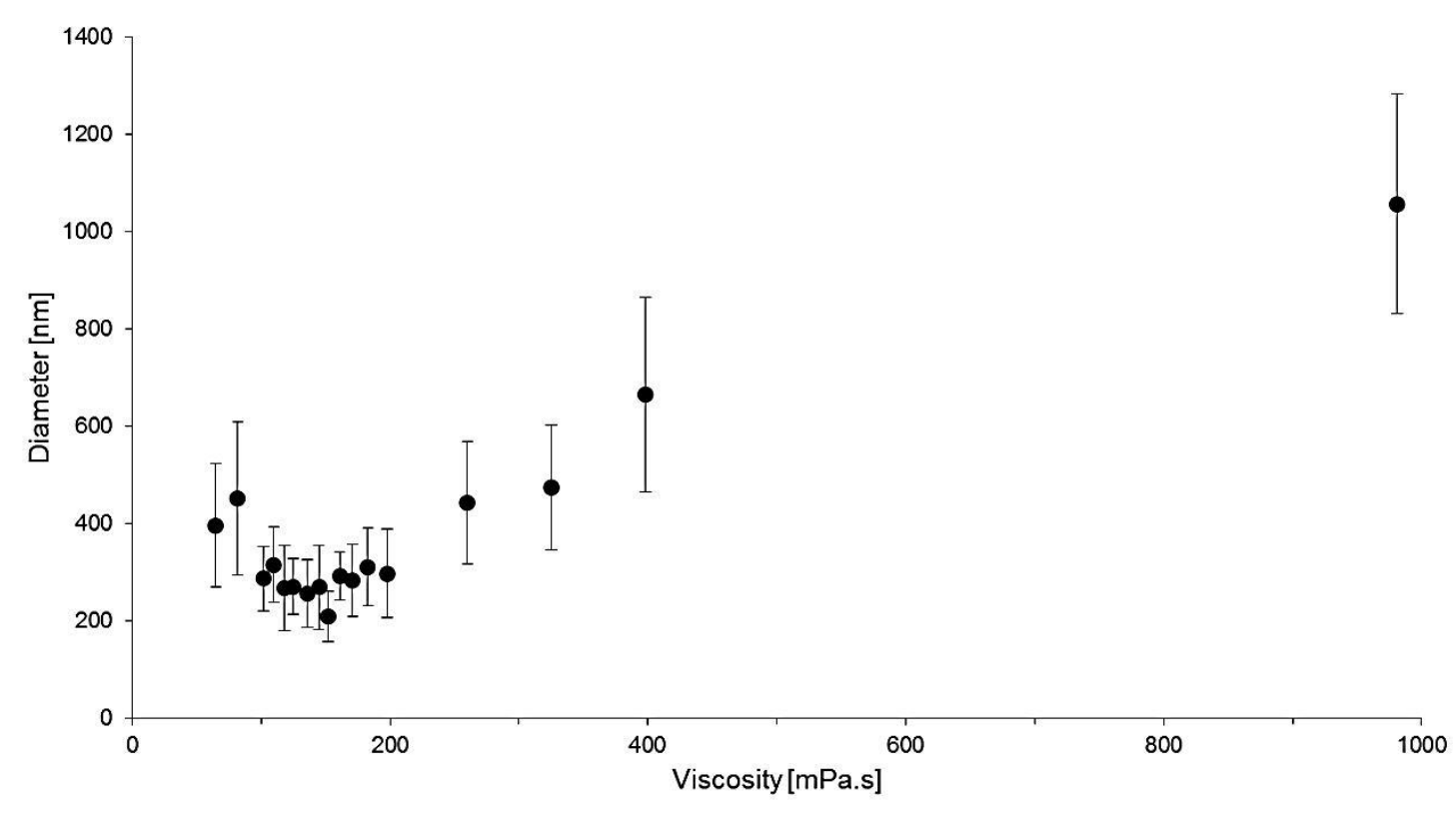

Fig. 4 Fibre diameters of silica nanofibres as a function of the sol viscosity

In addition the reproducibility within the stable electrospinning region was guaranteed, as demonstrated by the constant diameter of two samples with the same viscosity (135 mPa.s and $136 \mathrm{mPa} . \mathrm{s})$. The samples showed a diameter of $270 \pm 70 \mathrm{~nm}$ and $260 \pm 70 \mathrm{~nm}$.

It should, however, be noted that the stable electrospinning of silica nanofibres showed to be more difficult compared to the electrospinning of certain polymer nanofibres, where a stable Taylor cone in time could be obtained. An instability of 
the Taylor cone after a time period was always noticed during electrospinning of the silica nanofibres, however, the stability of the Taylor cone recovered after removal of the solidified piece.

As stated above the viscosity is caused by the evaporation of ethanol, a change in relative concentrations of the components and most importantly by the degree of crosslinking. Therefore, the spinnability of the silica sols is determined as well by this degree of crosslinking. A trend of the change in condensation degree with a change in viscosity of the sols is given by FTIR spectroscopy. The FTIR spectra of silica nanofibres obtained from sols having varying viscosities are shown in Figure 5. All samples show a broad peak near $3300 \mathrm{~cm}^{-1}$ which is assigned to $\mathrm{OH}$ vibrations. The peaks at $1070 \mathrm{~cm}^{-1}$ and $795 \mathrm{~cm}^{-1}$ are assigned to Si-O-Si vibrations and the peak at $952 \mathrm{~cm}^{-1}$ can be assigned to $\mathrm{Si}-\mathrm{OH}$ stretch. To examine the influence of the sol viscosity and thus the condensation degree, the FTIR spectra of all samples were normalized on the largest peak, namely the peak at $1070 \mathrm{~cm}^{-1}$ attributed to $\mathrm{Si}-\mathrm{O}-\mathrm{Si}$ vibrations. A clear decrease of the $\mathrm{Si}-\mathrm{OH}$ stretch peak at 952 $\mathrm{cm}^{-1}$ can be seen, Figure 6, with increasing viscosity of the sols used to electrospin the nanofibres. This indicates that the condensation degree is an important parameter probably influencing the electrospinning process.

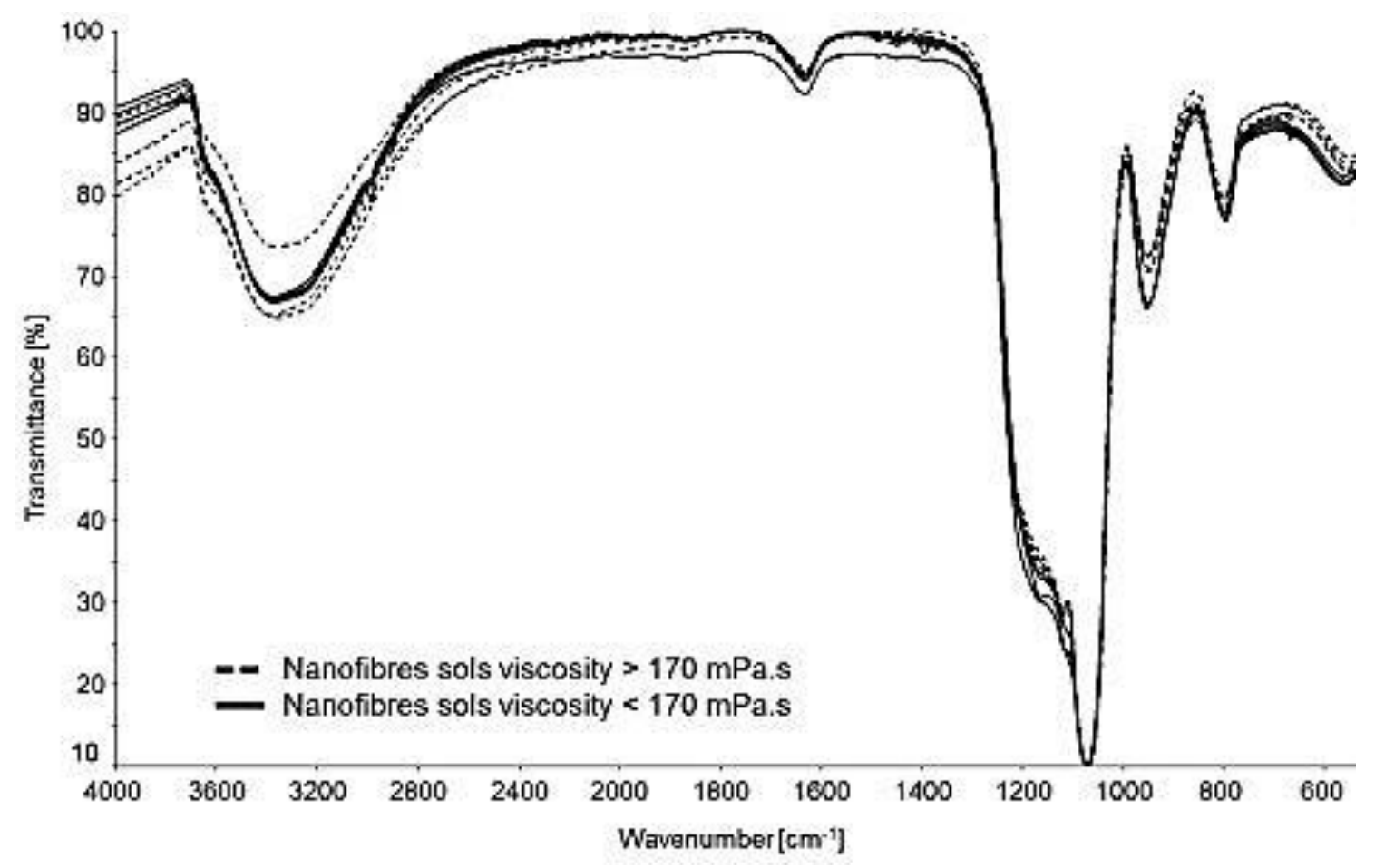

Fig. 5 FTIR spectra nanofibres obtained from sols having varying viscosities 


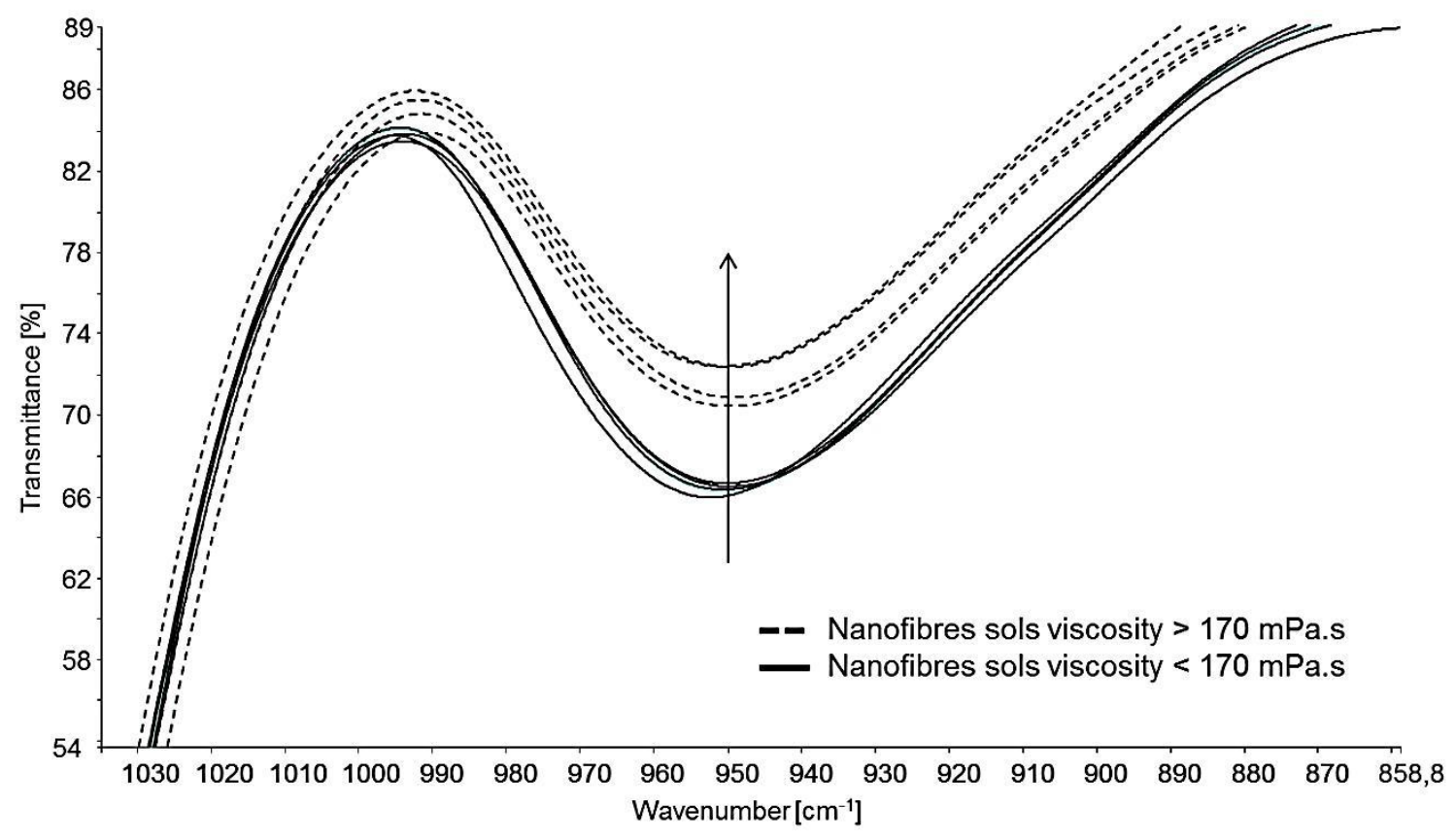

Fig. 6 Detail of Si-OH peak at $952 \mathrm{~cm}^{-1}$ of FTIR spectra silica nanofibres

\section{Effect of diluting sol to desired viscosity}

Dilution of a sol having a viscosity that exceeds the stable viscosity window may aid to an easy sample preparation since a specific viscosity and thus a specific fibre diameter can be easily obtained. However, as mentioned above the initial degree of crosslinking may have an influence. To analyse this, TEOS sols were prepared with a higher viscosity than the desired one. These sols were diluted with ethanol to obtain viscosities around the optimal viscosities being 120-200 mPa.s. The different samples obtained with this method are summarized in Table 2. All samples were electrospinnable, but the Taylor cone was stable for a shorter period of time compared to the electrospinning of freshly prepared sols. This less stable electrospinning might be attributed to a too high degree of crosslinking. Indeed, due to heating, ethanol evaporates, the relative concentrations of the components change and the sol starts to crosslink, thus increasing the viscosity. Once a certain degree of crosslinking is obtained, dilution of the sol with ethanol reduces the viscosity, but the degree of crosslinking is not affected, causing unstable electrospinning. This hypothesis is confirmed by the electrospinnability of sample 5 and 6 which are shown in Figure 7 (a,b). These samples with a viscosity of 114 $\mathrm{mPa} . \mathrm{s}$ and $115 \mathrm{mPa} . \mathrm{s}$ are diluted from solutions with a viscosity of respectively $1340 \mathrm{mPa} . \mathrm{s}$ and $562 \mathrm{mPa} . \mathrm{s}$. At a viscosity of $115 \mathrm{mPa} . \mathrm{s}$ uniform nanofibres were 
obtained starting from a fresh sol, although being at the edge of the stable electrospinning region. No uniform nanofibres were observed working with a diluted sol (Figure $7(a, b)$ ). Furthermore, a clear influence of the viscosity of the original sol is noticed. Beads are much larger for the sample prepared from a solution of $1340 \mathrm{mPa} . \mathrm{s}$ (Figure 7 (a)). The mean fibre diameter is similar for both samples, namely $365 \pm 70 \mathrm{~nm}$. It should be noted that beads were not taken into account for diameter determination.

Sample 8 and 9 with viscosities of respectively $138 \mathrm{mPa} . \mathrm{s}$ and $160 \mathrm{mPa} . \mathrm{s}$ prepared from sols with viscosities of respectively $969 \mathrm{mPa} . \mathrm{s}$ and $200 \mathrm{mPa} . \mathrm{s}$, have viscosities in the stable electrospinning viscosity range. However, both samples could not be electrospun in a stable manner. At a high magnification, Figure 5 $(\mathrm{c}, \mathrm{e})$, uniform nanofibres are visible for both samples. However, when the magnification is decreased (Figure $7(\mathrm{~d}, \mathrm{f})$ ) it is clearly visible that beads are noticed for sample 8 prepared at a higher viscosity while these are absent in sample 9. This confirms again the clear influence and importance of the viscosity of the original sol and more importantly of the initial degree of crosslinking of the sol.

Table 2 Overview samples obtained by dilution of fresh sols

\begin{tabular}{llll}
\hline Sample $^{\circ}$ & Viscosity (mPa.s) & $\begin{array}{l}\text { Viscosity } \\
\text { TEOS sol } \\
\text { (mPa.s) }\end{array}$ & $\begin{array}{l}\text { original } \\
(1 \text { to 5) }\end{array}$ \\
\hline 1 & 92 & 371 & 3 \\
2 & 100 & 358 & 3 \\
3 & 105 & 1025 & 3 \\
4 & 110 & 137 & 3 \\
5 & 114 & 1340 & 3 \\
6 & 115 & 562 & 3 \\
7 & 131 & 372 & 3 \\
8 & 138 & 969 & 3 \\
9 & 160 & 200 & 3 \\
\hline
\end{tabular}




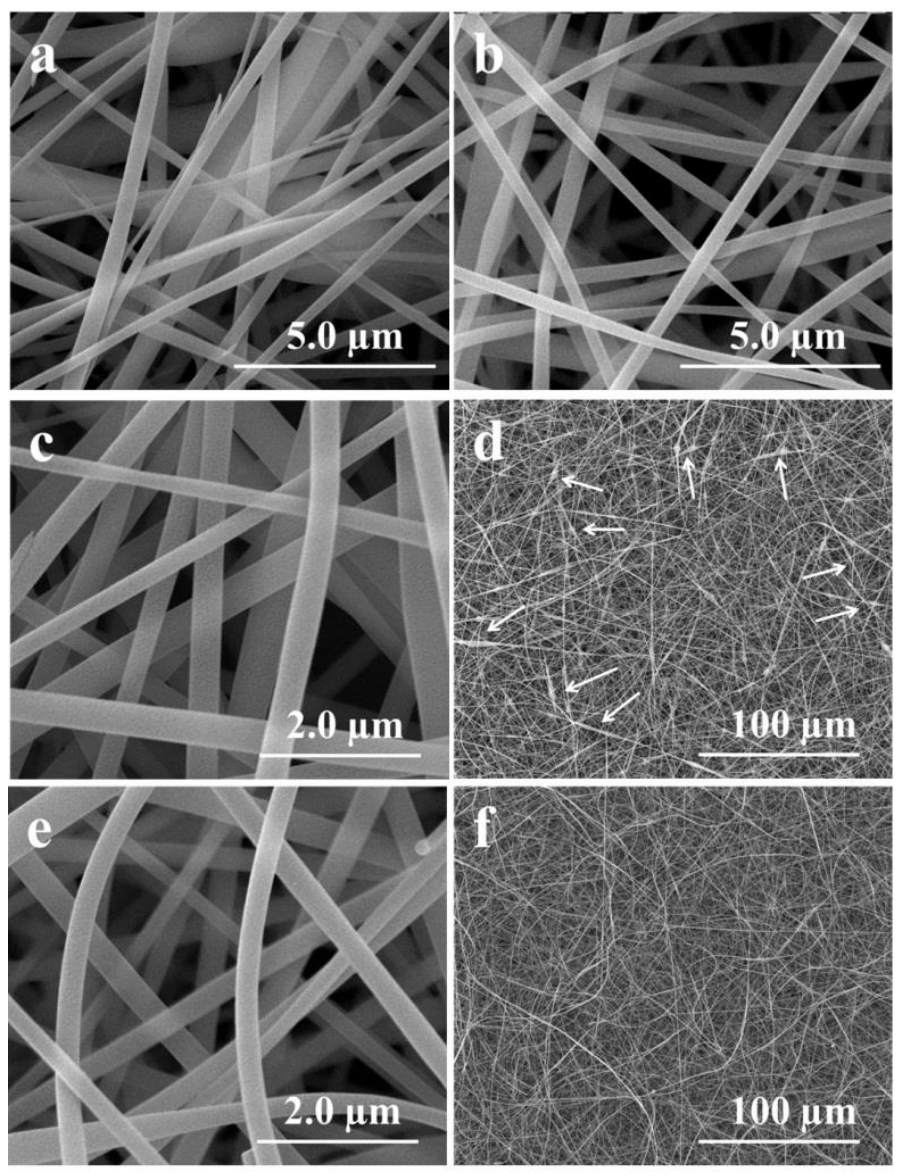

Fig. 7 SEM images of silica nanofibres obtained from diluted sols (a:114 mPa.s, b: 115 mPa.s, c and d:138 mPa.s, e and f:160 mPa.s)

The diameters of the nanofibres obtained from the diluted sols are more or less in the same range as those from the fresh sols as can be seen in Figure 8. A small increase in nanofibre diameter can, however, be noticed. Coefficient of variations are typically between $20 \%$ and $30 \%$, analogous to the coefficients of variation for nanofibres prepared from fresh sols, but as stated above, beads were not taken into account for nanofibre diameter determination. Electrospinning of diluted sols is thus possible, however, not as stable as electrospinning of fresh sols. In the stable region beadless and uniform nanofibres can be obtained if the viscosity of the original sol falls within the desired viscosity range. 


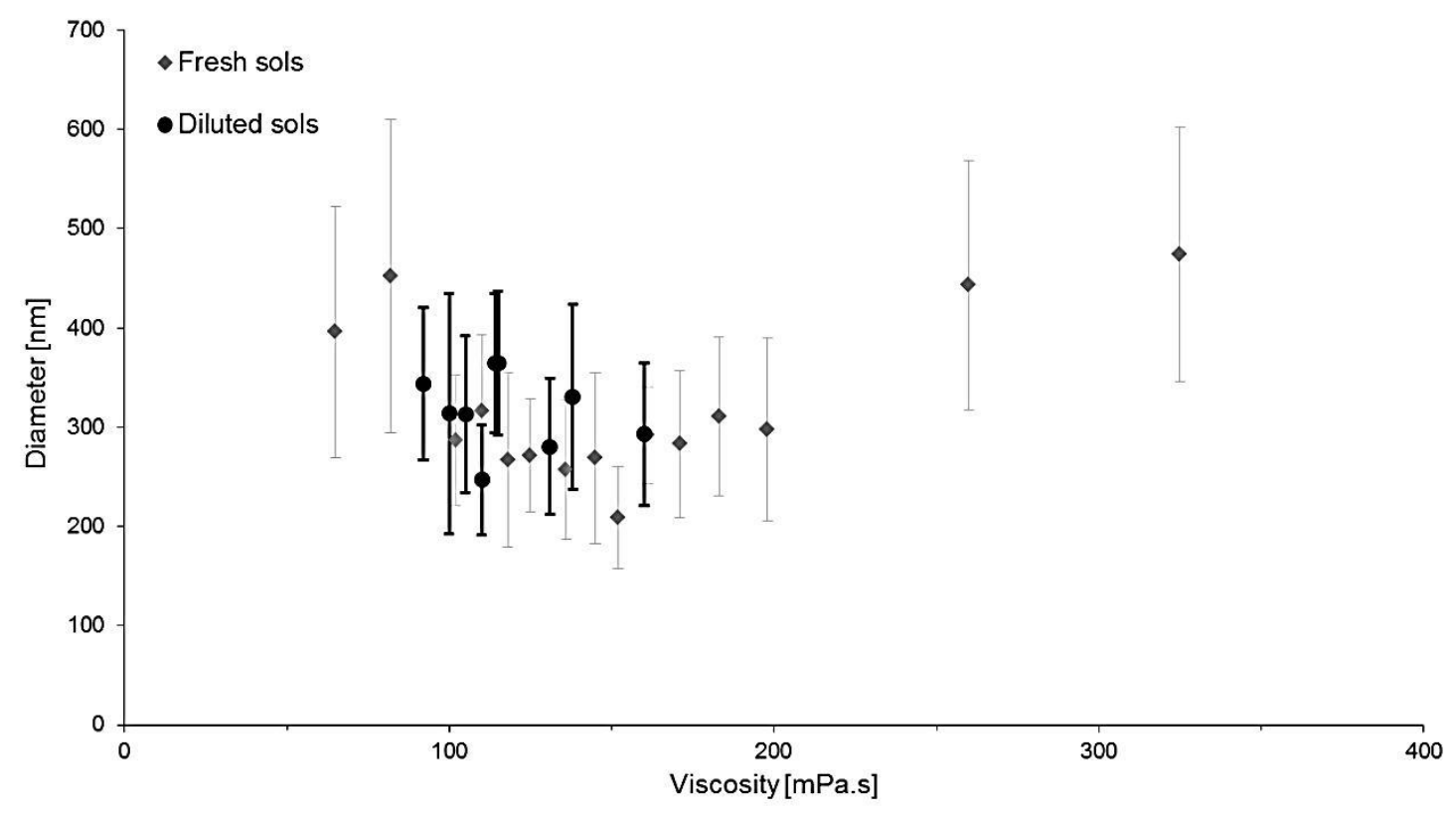

Fig. 8 Fibre diameters of silica nanofibres obtained from fresh and diluted sols as a function of the sol viscosity

\section{Conclusions}

In this paper, electrospinning of TEOS sols with different viscosities was evaluated. Different stability regions, having a varying spinnability could be determined. A clear viscosity region could be noticed for which electrospinning of TEOS sols was stable, namely between 120 and 200 mPa.s. Furthermore, electrospinning of sols having viscosities in this region resulted in nanofibres with the finest mean nanofibre diameter. To obtain these desired viscosities dilution of sols with ethanol was possible as well. To this end, the electrospinning of freshly prepared TEOS sols was compared to the electrospinning of diluted sols, which were obtained from fresh sols with viscosities above the stable viscosity region. The electrospinning process of the diluted sols was less stable, probably caused by the high degree of crosslinking of the original sol. However, uniform nanofibres could be obtained. Nonetheless, the optimum viscosity for stable electrospinning was thus ideally obtained by preparing a new sol. We can thus conclude that not only the viscosity and the relative concentrations determine the spinnability of the siloxane sols, but the degree of crosslinking has a major influence as well. Further research will focus on a more detailed characterization of this degree of crosslinking. 


\section{Acknowledgement}

Special gratitude towards the Agency for Innovation by Science and Technology (IWT) since they funded the research by a Ph.D. grant.

\section{References}

[1] Greiner A, Wendorff JH. (2007) Angew. Chem. Int. Ed. 46:5670-5703

[2] Wendorff JH, Agarwal S, Greiner A (2012) Electrospinning. Materials, Processing and Applications. Wiley-VCH, Germany

[3] Ramakrishna S, Fujihara K, Teo WE, Lim TC, Ma Z (2005) Electrospinning and nanofibres. World Scientific publishing, Singapore

[4] De Schoenmaker B, Goethals A, Van der Schueren L, Rahier H, De Clerck K (2012) J Mater Sci 47:4118-4126

[5] Van der Schueren L, De Schoenmaker B, Kalaoglu O, De Clerck K (2011) Eur. Polym. J 47:1256-1263

[6] De Vrieze S, Westbroek P, Van Camp T, De Clerck K (2010) J. Appl. Polym. Sci 115:837-842

[7] De Vrieze S, De Schoenmaker B, Ceylan O, Depuydt J, Van Landuyt L, Rahier H, Van Assche G, De Clerck K (2011) J. Appl. Polym. Sci. 119:2984-2990

[8] Yamaguchi T, Sakai S, Kawakami K (2008) J. Sol-Gel Sci. Technol. 48:350-355

[9] Sakai S, Yamaguchi T, Putra RA, Watanabe R, Kawabe M, Taya M, Kawakami K (2012) J. Sol-Gel Sci. Technol. 61:374-380

[10] Wu S, Li F, Wu Y, Xu R, Li G (2010) Chem. Commun. 46:1694-1696

[11] Teng M, Wang H, Li F, Zhang B (2011) J. Colloid Interface Sci. 335:23-28

[12] Chen Q, Zhang L, Yoon MK, Wu XF, Arefin RH, Fong H (2012) J. Appl. Polym. Sci. $124: 444-451$

[13] Chen Q, Zhang L, Zhao Y, Wu XF, Fong H (2012) Composites: Part B 43:309-316

[14] Wang X, Fang H, Ren P, Yu H, Li J (2012) Mater. Res. Bull. 47:1734-1739

[15] Chen LJ, Liao JD, Lin SJ, Chuang YJ, Fu YS (2009) Polymer 50:3516-3521

[16] Roh SH, Lee YA, Lee YW, Kim SI (2008) J. Nanosci. Nanotechnol. 8:5147-5151

[17] Shao C, Kim H, Gong J, Lee D (2002) Nanotechnology 13:635-637

[18] Liu Y, Sagi S, Chandrasekar R, Zhang L, Nyle E, Fong H (2008) J. Nanosci. Nanotechnol. $8: 1528-1536$

[19] Lee SW, Kim YU, Choi SS, Park TY, Joo YL, Lee SG (2007) Mater. Lett. 61:889-893

[20] Iimura K, Oi T, Suzuki M, Hirota M (2010) Adv. Powder Technol. 21:64-68

[21] Choi SS, Lee SG, Im SS, Kim SH, Joo YL (2003) J. Mater. Sci. Letters 22:891-893

[22] Choi SS, Chu B, Lee SG, Lee SW, Im SS, Kim SH, Park JK (2004) J. Sol-Gel Sci. Technol. 30: $215-221$

[23] Xu Y, Zhou W, Zhang L, Cheng L (2000) J. Mater. Process. Technol. 101:44-46

[24] Huang ZM, Zhang YZ, Kotaki M, Ramakrishna S (2003) Compos. Sci. Technol. 63:22232253 
[25] Mit-uppatham C, Nithitanakul M, Supaphol P (2004) Macromol. Chem. Phys. 205:2327-2338

[26] Jacobs V, Anandjiwala RD, Maaza M (2010) J. Appl. Polym. Sci. 115: 3130-3136

Fig. 1 Spinnability as a function of the sol viscosity

Fig. 2 Stable Taylor cone (a) and unstable Taylor cone (b)

Fig. 3 SEM images electrospun silica nanofibres (a: $65 \mathrm{mPa} . \mathrm{s}, \mathrm{b}: 102 \mathrm{mPa} . \mathrm{s}, \mathrm{c}: 110 \mathrm{mPa} . \mathrm{s}, \mathrm{d}: 125$ mPa.s, e:145 mPa.s, f:198 mPa.s, g:398 mPa.s, h:981 mPa.s)

Fig. 4 Fibre diameters of silica nanofibres as a function of the sol viscosity

Fig. 5 FTIR spectra nanofibres obtained from sols having varying viscosities

Fig. 6 Detail of Si-OH peak at $952 \mathrm{~cm}^{-1}$ of FTIR spectra silica nanofibres

Fig. 7 SEM images of silica nanofibres obtained from diluted sols (a:114 mPa.s, b: $115 \mathrm{mPa} . \mathrm{s}, \mathrm{c}$ and d:138 mPa.s, e and f:160 mPa.s)

Fig. 8 Fibre diameters of silica nanofibres obtained from fresh and diluted sols as a function of the sol viscosity

Table 1 Explanation of the different spinnabilities

Table 2 Overview samples obtained by dilution of fresh sols 